\title{
Formal Analysis of the Cauchy Problem for a System Associated with the (2+1)-Dimensional Krichever-Novikov Equation
}

\author{
Werner M. Seiler \\ Institut für Algorithmen und Kognitive Systeme, Universität Karlsruhe \\ D-76128 Karlsruhe, Germany \\ Peter J. Vassiliou \\ Faculty of Information Science and Engineering, University of Canberra \\ Belconnen ACT 2616, Australia \\ and \\ Colin Rogers \\ School of Mathematics, University of New South Wales \\ Sydney 2052, NSW, Australia
}

\begin{abstract}
The singularity manifold equation of the Kadomtsev-Petviashvili equation, the socalled Krichever-Novikov equation, has an exact linearization to an overdetermined system of partial differential equations in three independent variables. We study in detail the Cauchy problem for this system as an example for the use of the formal theory of differential equations. A general existence and uniqueness theorem is established. Formal theory is then contrasted with Janet-Riquier theory in the formulation of Reid. Finally, the implications of the results for the Krichever-Novikov equation are outlined.
\end{abstract}

Mathematics Subject Classification (1991): 35N05, 35C10, 35A07

Key words: Krichever-Novikov equation, Cauchy problem, existence and uniqueness theorem, formal theory, Janet-Riquier theory

\section{Introduction}

In recent articles $[1,2]$ particular initial/boundary value problems on the halfplane and quarter-plane have been considered for the $(2+1)$-dimensional KricheverNovikov equation

$$
-3\left(\frac{\phi_{y}}{\phi_{x}}\right)_{y}+\frac{\partial}{\partial x}\left[\frac{\phi_{t}}{\phi_{x}}-\{\phi ; x\}-\frac{3}{2}\left(\frac{\phi_{y}}{\phi_{x}}\right)^{2}\right]=0
$$

with the Schwarzian derivative $\{\phi ; x\}=\partial_{x}\left(\phi_{x x} / \phi_{x}\right)-1 / 2\left(\phi_{x x} / \phi_{x}\right)^{2}$ via the particular linear decomposition

$$
\phi_{y}+\phi_{x x}=0, \phi_{t}-4 \phi_{x x x}=0
$$

to the backward heat and the linearized Korteweg-de Vries equation. The Krichever-Novikov equation is important in that it represents the singularity manifold 
equation of the Kadomtsev-Petviashvili equation as derived from the Painlevé analysis of the latter. Moreover, it is itself integrable. Further, it can be linked to the integrable Harry-Dym equation via a reciprocal transformation [3].

It was the latter connection which was originally used in Ref. [1] to obtain the above decomposition. Thus it was shown in Ref. [4] that the (2+1)-dimensional Harry-Dym equation

$$
\rho_{t}+\rho^{-1}\left(\rho^{-1}\right)_{x x x}-3 \rho^{3}\left(\rho^{-2} \partial_{x}^{-1} \rho_{y}\right)_{y}=0
$$

is invariant under the transformation

$$
\begin{gathered}
d x^{\prime}=\frac{\rho}{\rho^{\prime}} d x+\left(\frac{1}{\rho \rho^{\prime}}\right)_{x} d y-2\left(\frac{\rho_{x}}{\rho^{\prime} \rho^{3}}+\frac{2 \rho_{x}^{\prime}}{\left(\rho \rho^{\prime}\right)^{2}}+\frac{3 \partial_{x}^{-1} \rho_{y}}{\rho \rho^{\prime}}\right)_{x} d t \\
y^{\prime}=y, t^{\prime}=t
\end{gathered}
$$

The compatibility conditions for this reciprocal transformation are

$$
\begin{gathered}
(\psi \rho)_{y}=\left(\psi \rho^{-1}\right)_{x} x \\
(\psi \rho)_{t}=\left[\left(\rho^{-2}\right)_{x} \psi+4 \rho^{-2} \psi_{x}-6 \rho^{-1}\left(\partial_{x}^{-1} \rho_{y}\right) \psi\right]_{x x} \\
(\psi \rho)_{x} t=\left[\left(\rho^{-2}\right)_{x} \psi+4 \rho^{-2} \psi_{x}-6 \rho^{-1}\left(\partial_{x}^{-1} \rho_{y}\right) \psi\right]_{x y}
\end{gathered}
$$

where $\psi=\left(\rho^{\prime}\right)^{-1}$ provides a linear representation for (3).

In Ref. [3] it was established that the $(2+1)$-dimensional Harry-Dym equation (3) is reciprocally linked to (1) via a transformation $H$. (3) expressed in $\left(x^{\prime}, y^{\prime}, t^{\prime}\right)$ admits the solution $\rho^{\prime}=1$. Hence, the compatibility conditions (5) show that $\rho(x, y, t)$ is a solution of $(3)$, if it is a simultaneous solution of the nonlinear heat equation

$$
\rho_{y}=\left(\frac{1}{\rho}\right)_{x x}
$$

together with

$$
\rho_{t}=-2\left(\frac{1}{\rho^{2}}\right)_{x x x}
$$

and

$$
\left(\frac{1}{\rho}\right)_{x t}=-2\left(\frac{1}{\rho^{2}}\right)_{x x y} .
$$

It is seen that the latter condition is automatically implied by (6) and (7), so that it may be discarded. Applications to (6) and (7) of the transformation $H$ as given in Ref. [3] produces the simultaneous linearization (2) of these equations. Accordingly, if $\phi$ is a simultaneous solution of the linear equations (2), then it is also a solution of the $(2+1)$-dimensional Krichever-Novikov equation (1). 
This result may be obtained alternatively from the linear eigenfunction representation for the Kadomtsev-Petviashvili equation. In Ref. [2] the linear decomposition was used to obtain solutions to classes of initial/boundary value problems on the half-planes $-\infty<x<\infty, y \leq 0$ and $-\infty<y<\infty, x \geq 0$ as well as on the quarter-plane $x \geq 0, y \leq 0$.

It is noted that analogous decompositions can readily be obtained for eigenfunction equations associated with other $(2+1)$-dimensional integrable systems such as the $(2+1)$-dimensional Sawada-Kotera equation $[5,6]$. Certain boundary value problems may then be solved after the manner of Refs. [1, 2].

In the present paper, by contrast, our concern is with pure initial value problems for an overdetermined pair of linear equations of the above type, namely

$$
u_{t}=u_{x x x}, u_{y}=u_{x x} .
$$

Importantly, the linear decomposition result shows that such solutions as one obtains apply 'mutatis mutandis' to initial value problems for the integrable $(2+1)$ dimensional Krichever-Novikov equation. It is recalled that initial value problems for nonlinear integrable equations in $1+1$ dimensions are commonly treated by the Inverse Scattering Transform and more recently by the $\bar{\partial}$-Method in $2+1$ dimensions. The connections between the results obtained in the present paper by formal analysis and those derivable by variants of the Inverse Scattering Method remain to be explored.

We use the formal theory of partial differential equations [7] to show that the analytic solution space of system (9) can be parameterized by three functions of one variable. This fact is exploited to solve Cauchy problems for the system.

This formal analysis is necessary, because for non-normal systems, i.e. systems not satisfying the conditions of the Cauchy-Kowalevsky theorem, it is not trivial to deduce how much Cauchy data can be prescribed in order to render the problem well posed. This holds especially for overdetermined systems like (9). It turns out that involution provides the key [8].

The analysis is further used to highlight differences between the notion of involution in formal theory and the notion of a passive system in Janet-Riquier theory $[9,10]$. This theory is especially useful for the construction of formally wellposed initial value problems [11], i.e. problems where exactly the correct amount of Cauchy data is prescribed to guarantee existence and uniqueness.

The paper is organized as follows: After a brief introduction into the theoretical framework of formal analysis, Section 3 will treat system (9) in detail. After the construction of some typical power series solutions, we will consider in Section 5 the general Cauchy problem for our system. The last section compares the approach taken here with the Janet-Riquier theory. 


\section{Formal Theory of Differential Equations}

Formal theory uses a geometric approach to differential equations based on the jet bundle formalism. It is beyond the scope of this paper to give a detailed introduction to the underlying theory. The reader is referred to the literature $[7,12,13]$. Here we are concerned with two topics: the definition of an involutive system and how to compute the arbitrariness of the general solution of such a system.

We will always work in a local coordinate system, although the whole theory can be expressed in a coordinate independent way. Let $x_{1}, \ldots, x_{n}$ denote the independent and $u^{1}, \ldots, u^{m}$ the dependent variables. Derivatives are written in multi-index notation $p_{\mu}^{\alpha}=\partial^{|\mu|} u^{\alpha} / \partial x_{1}^{\mu_{1}} \cdots \partial x_{n}^{\mu_{n}}$ where $|\mu|=\mu_{1}+\cdots+\mu_{n}$ is the length of $\mu=\left[\mu_{1}, \ldots, \mu_{n}\right]$. The derivatives up to order $q$ define a local section for the $q$-th order jet bundle $\mathcal{J}_{q}$. A differential equation $\mathcal{R}_{q}$ of order $q$ can then be described locally by a system of equations

$$
\mathcal{R}_{q}: \Phi^{\tau}\left(x_{i}, u^{\alpha}, p_{\mu}^{\alpha}\right)=0, \quad \tau=1, \ldots, p ;|\mu| \leq q .
$$

Geometrically, it can be regarded as a fibered submanifold of $\mathcal{J}_{q}$.

At least some of the ideas behind the concept of involution can best be understood by considering the construction of a formal power series solution for the system order by order. We will describe a method that works for any system independent of whether it can be solved for some derivatives or not. For this purpose, we introduce the symbol $\mathcal{M}_{q}$ of a system $\mathcal{R}_{q}$.

DEFINITION 1. The symbol $\mathcal{M}_{q}$ of the system (10) is the solution space of the linear system of (algebraic!) equations

$$
\mathcal{M}_{q}: \sum_{\alpha,|\mu|=q}\left(\frac{\partial \Phi^{\tau}}{\partial p_{\mu}^{\alpha}}\right) v_{\mu}^{\alpha}=0 .
$$

(By abuse of language, we will refer to both the linear system and its solution space as the symbol).

Here $v_{\mu}^{\alpha}$ are coordinates of a finite vector space, i.e. we introduce one coordinate for each derivative of order $q$. Definition 1 is most easily understood by considering a quasi-linear system, i.e. a system which is linear in the derivatives $p_{\mu}^{\alpha}$ with $|\mu|=q$. For such a system the symbol is simply obtained by taking only the highest order part and substituting $v_{\mu}^{\alpha}$ for $p_{\mu}^{\alpha}$.

We make a power series ansatz for the general solution of the differential equation $\mathcal{R}_{q}$ by expanding it around some point $x^{0}$. Then we substitute this ansatz into the equations (10) and evaluate at $x^{0}$. This yields a system of algebraic equations for the Taylor coefficients up to order $q$. The remaining coefficients can be computed by linear algebra only. 
For the coefficients of order $q+r$ we use the prolonged systems $\mathcal{R}_{q+r}$ which are obtained by differentiating each equation in $\mathcal{R}_{q} r$ times totally with respect to all independent variables. They are all quasi-linear. If we substitute again the power series ansatz into the prolonged system $\mathcal{R}_{q+r}$ and evaluate at $x^{0}$, we get an inhomogenous linear system for the derivatives of order $q+r$. Its homogeneous part is given by the prolonged symbol $\mathcal{M}_{q+r}$, i.e. the symbol of $\mathcal{R}_{q+r}$.

The coefficients of lower order appear in the coefficients and in the right hand side of this linear system. Thus we are able to express the coefficients of order $q+r$ through the coefficients of lower order. This is the precise meaning of constructing a power series order by order. The arbitrariness of the general solution is now reflected by the dimensions of the prolonged symbols, because at each order $\operatorname{dim} \mathcal{M}_{q+r}$ coefficients are not determined by the differential equations but can be chosen freely.

This construction will, however, fail, if there are non-trivial integrability conditions, i.e. equations of order $q+r$ which are functionally independent of the equations contained in the prolonged system $\mathcal{R}_{q+r}$ and which are satisfied by every solution of the system. Such equations arise usually by cross-differentiating and are detected only in some higher prolongation. They pose additional conditions on the coefficients of order $q+r$. Hence they must all be known to pursue the above described procedure. We call a system which contains all its integrability conditions a formally integrable system.

For formally integrable systems it is thus possible to construct order by order a formal power series solution. In many applications it is of interest to know the number of arbitrary Taylor coefficients at any order, i.e. $\operatorname{dim} \mathcal{M}_{q+r}$ for all $r$. Formal integrability does, however, not suffice to determine these dimensions in advance.

For this purpose, we introduce the class of a multi-index $\mu=\left[\mu_{1}, \ldots, \mu_{n}\right]$. It is the smallest $k$ for which $\mu_{k}$ is different from zero. If we consider the symbol (11) as a matrix, then its columns are numbered by the coordinates $v_{\mu}^{\alpha}$. We can order them by class, i.e. taking always a column with a multi-index of higher class left of one with lower class. Then we can compute a row echelon form.

In this so-called solved form the symbol is especially easy to analyze. Since we need only linear operations to obtain it, we can always perform the same operations with the whole system $\mathcal{R}_{q}$ and thus assume that (11) yields the symbol directly in solved form. We denote the number of rows where the leading entry is of class $k$ by $\beta_{q}^{(k)}$ and we associate with each such row the multiplicative variables $x_{1}, \ldots, x_{k}$.

It is now easy to see that if we prolong each equation only with respect to its multiplicative variables, we obtain independent equations, because each equation will have a different leading term. The question is now, whether prolongation with respect to the non-multiplicative variables leads to additional independent equations. If not, we call the symbol involutive. 
DEFINITION 2. The symbol $\mathcal{M}_{q}$ is called involutive, if

$$
\operatorname{rank} \mathcal{M}_{q+1}=\sum_{k=1}^{n} k \beta_{q}^{(k)} .
$$

The system $\mathcal{R}_{q}$ is called involutive, if it is formally integrable and its symbol is involutive.

The above definition of the $\beta_{q}^{(k)}$ is obviously coordinate dependent and it seems, as if the involution of a symbol depends on the chosen coordinate system. But one can show that almost every coordinate system leads to the same values for the $\beta_{q}^{(k)}$. These are characterized by the property that $\beta_{q}^{(n)}, \beta_{q}^{(n-1)}+\beta_{q}^{(n)}, \ldots, \sum \beta_{q}^{(k)}$ are maximal. A coordinate system which leads to these values is called $\delta$-regular. Definition 2 assumes that the $\beta_{q}^{(k)}$ are computed in such a coordinate system. (Another approach to this problem was presented in Ref. [11]).

It is now an important property of involutive symbols that their prolongations are again involutive. Since prolonging an equation with respect to one of its multiplicative variables $x_{i}$ yields an equation of class $i$, we get $\beta_{q+1}^{(i)}=\sum_{k=i}^{n} \beta_{q}^{(k)}$. Inductive use of this relation leads to

$$
\beta_{q+r}^{(k)}=\sum_{i=k}^{n}\left(\begin{array}{c}
r+i-k-1 \\
r-1
\end{array}\right) \beta_{q}^{(i)}
$$

and thus the searched for expression for the ranks of the prolonged symbols

$$
\operatorname{rank} \mathcal{M}_{q+r}=\sum_{k=1}^{n}\left(\begin{array}{c}
r+k-1 \\
r
\end{array}\right) \beta_{q}^{(k)} .
$$

Besides the possibility to predict the number of arbitrary Taylor coefficients at any order, involutive systems have another important property. There exist an easily applicable criterion to check whether a system is involutive or not. The problem of the definition of formal integrability is to prove that a system does not generate non-trivial integrability conditions at any prolongation order. This can, however, be done for systems with an involutive symbol.

THEOREM 3. Let $\mathcal{R}_{q}$ be a $q$-th order differential equation with an involutive symbol $\mathcal{M}_{q}$. If there arise no integrability conditions during the prolongation of $\mathcal{R}_{q}$ to $\mathcal{R}_{q+1}$, then $\mathcal{R}_{q}$ is involutive.

Whether or not integrability conditions arise during the prolongation, can be seen from a comparison of the dimensions of the submanifolds. For an involutive system the equation

$$
\operatorname{dim} \mathcal{R}_{q}=\operatorname{dim} \mathcal{R}_{q+1}-\operatorname{dim} \mathcal{M}_{q+1}
$$


must hold. It shows that integrability conditions are always connected with a rank deficit in the symbol.

According to the Cartan-Kuranishi Theorem, any system can be completed to an involutive one by a finite number of prolongations and projections (i.e. addition of integrability conditions). Refs. [14, 15] describe an algorithm for this completion and its implementation in the computer algebra system AXIOM.

\section{Application to our System}

To apply the formalism described in the last section to system (9), it is convenient to rewrite it as a second-order system in order to embed it in the jet bundle $\mathcal{J}_{2}$. This is achieved by inserting the second equation of (9) into the first one to get $u_{t}=u_{x y}$. Thus we obtain the system

$$
\mathcal{R}_{2}:\left\{\begin{array}{l}
u_{x y}-u_{t}=0 \\
u_{x x}-u_{y}=0
\end{array}\right.
$$

This system is, however, not in involution. It is not even formally integrable, as there exists clearly the integrability condition $u_{y y}-u_{x t}=0$.

PROPOSITION 4. The second-order system

$$
\mathcal{R}_{2}^{(1)}:\left\{\begin{array}{l}
u_{x y}-u_{t}=0 \\
u_{x x}-u_{y}=0 \\
u_{y y}-u_{x t}=0
\end{array}\right.
$$

is involutive and equivalent to the original system (9).

Proof. To apply Theorem 3, we must first check whether the symbol $\mathcal{M}_{2}^{(1)}$ of this system is involutive. Definition 1 yields

$$
\mathcal{M}_{2}^{(1)}: \begin{cases}v_{x y} & =0 \\ v_{x x} & =0 \\ v_{y y}-v_{x t} & =0\end{cases}
$$

To compute the $\beta_{2}^{(k)}$ we need an ordering of the independent variables. We choose $x_{1}=t, x_{2}=y, x_{3}=x$. Then we see that the second equation in (18) is of class 3 and we get the multiplicative variables $(t, y, x)$. The other equations are both of class 2 and yield the multiplicative variables $(t, y)$. Hence, we obtain $\beta_{2}^{(1)}=0, \beta_{2}^{(2)}=$ $2, \beta_{2}^{(3)}=1$. Since this yields the maximal possible values for $\beta_{2}^{(3)}, \beta_{2}^{(2)}+\beta_{2}^{(3)}$ and $\beta_{2}^{(1)}+\beta_{2}^{(2)}+\beta_{2}^{(3)}$, the coordinate system is $\delta$-regular.

To use Definition 2 of an involutive symbol we need the prolonged system

$$
\mathcal{R}_{3}^{(1)}:\left\{\begin{array}{lll}
u_{x x y}-u_{x t}=0, & u_{x y y}-u_{y t}=0, & u_{x y t}-u_{t t}=0, \\
u_{x x x}-u_{x y}=0, & u_{x x y}-u_{y y}=0, & u_{x x t}-u_{y t}=0, \\
u_{x y y}-u_{x x t}=0, & u_{y y y}-u_{x y t}=0, & u_{y y t}-u_{x t t}=0
\end{array}\right.
$$


and its symbol

$$
\mathcal{M}_{3}^{(1)}:\left\{\begin{array}{lll}
v_{x x y}=0, & v_{x y y}=0, & v_{x y t}=0, \\
v_{x x x}=0, & v_{x x y}=0, & v_{x x t}=0, \\
v_{x y y}-v_{x x t}=0, & v_{y y y}-v_{x y t}=0, & v_{y y t}-v_{x t t}=0 .
\end{array}\right.
$$

It is easy to see that this symbol has rank 7 . One can further see, that indeed all linearly independent equations are obtained by prolonging each equation of (17) with respect to its multiplicative variables only.

Thus the symbol $\mathcal{M}_{2}^{(1)}$ is involutive. All integrability conditions which arise by taking linear combinations of equations of $\mathcal{R}_{3}^{(1)}$ are already contained in $\mathcal{R}_{2}^{(1)}$. This can also be checked using Equation (15). We can thus conclude with Theorem 3 that $\mathcal{R}_{2}^{(1)}$ is an involutive system as claimed in the Proposition.

To show the equivalence of the two systems (9) and (17), we must compare their solution spaces. The addition of the integrability condition has no effect on the solution space, because every solution of the original system satisfies it. The change from a third-order system to a second-order system also makes no difference, as we are only interested in analytic solutions. Thus both systems have the same analytic solution space and can be considered as equivalent.

We have not shown here, how the system (17) can be deduced systematically starting with the system $\mathcal{R}_{2}$ and applying the algorithm presented in Refs. [14, 15], as the needed integrability condition is obvious. It is, however, worth mentioning, that this construction requires only linear algebra even for non-linear systems.

Finally, we compute the number of arbitrary functions in the general solution and the number of their argument. In general, there is no unique answer to this problem [8]. But here we are in a simple case. Since only arbitrary functions with one argument occur, it makes no difference whether they appear algebraically, as integrals or as derivatives.

PROPOSITION 5. Every representation of the general analytic solution of system (17) and thus of system (9) leading to a formal power series which can be constructed order by order depends on three arbitrary functions each of one argument.

Proof. As pointed out in Section 2, the arbitrariness can be measured by the number of parametric derivatives. It is given at each order by the dimension of the corresponding symbol. From the proof of Proposition 4 we know already that $\operatorname{dim} \mathcal{M}_{2}^{(1)}=\operatorname{dim} \mathcal{M}_{3}^{(1)}=3$. It is easy to see from (14) that all higher symbols must have exactly the same dimension. But constant dimension of all prolonged symbols can only occur, if all arbitrary functions have exactly one argument, because otherwise the dimension must grow. 


\section{Power Series Solutions}

The purpose of this section is to construct formal power series solutions for several initial value problems. This will shed some light on why we always stressed the order by order construction of the series. We start with the problem

$$
u\left(t, x_{0}, y_{0}\right)=h(t), \quad u_{x}\left(t, x_{0}, y_{0}\right)=h_{1}(t), \quad u_{y}\left(t, x_{0}, y_{0}\right)=h_{2}(t),
$$

where $x_{0}, y_{0}$ are arbitrary but fixed. We will not use the general method outlined in Section 2. It is more of theoretical than practical interest. Usually, one obtains power series solutions simpler by exploiting the special form of a given system.

We make the following ansatz for the series

$$
u(t, x, y)=u\left(t, x_{0}, y_{0}\right)+\left(x-x_{0}\right) u_{x}\left(t, x_{0}, y_{0}\right)+\left(y-y_{0}\right) u_{y}\left(t, x_{0}, y_{0}\right)+\cdots,
$$

i.e. we expand only with respect to the variables $x$ and $y$. Clearly we are finished, if we can express each derivative of the form

$$
u_{[p q]}:=\frac{\partial^{p+q} u}{\partial x^{p} \partial y^{q}}
$$

by $u, u_{x}, u_{y}$ and their $t$-derivatives. Four different cases arise

1. Suppose $p=0$. For $q=1$ we have already $u_{y}$. For $q=2$ the third equation of (17) says $u_{y y}=u_{x t}$. Thus we need here the integrability condition for the construction of the power series. Similarly, $u_{[03]}=u_{t x y}=u_{t t}$. For the higher values of $q$ we get a reduction to these lower values, as the last case showed that three $y$-derivatives can always be replaced by two $t$-derivatives.

2. Suppose $p-q=2 m, m \geq 0$. We first apply $q$ times the second equation of (17), then $m$ times the first one to get $u_{[p q]}=\partial^{p+m} u / \partial t^{p} \partial y^{m}$. But this is just a $t$-derivative of Case 1.

3. Suppose $p-q=2 m+1, m \geq 0$. As in the previous case, we obtain that $u_{[p q]}=\partial^{q+m} u / \partial t^{q+1} \partial y^{m-1}$ and thus a reduction to Case 1.

4. Suppose $p-q<0$. As in Case 2 , we can eliminate the $x$-derivatives and we obtain again a $t$-derivative of Case 1 with $q-p y$-derivatives.

Since each derivative in the Taylor series (22) is now expressed as $t$-derivative of one of the three functions $u, u_{x}, u_{y}$, it follows that each derivative can be expressed in terms of the Cauchy data $h, h_{1}, h_{2}$.

A closer inspection of the four cases shows that we have always expressed the derivative $u_{[p q]}$ by a derivative of the same or lower order. Thus the prerequisites of Proposition 5 are satisfied and we can conclude that the above constructed formal power series describes the general solution of (17) and thus also of (9) in a neighborhood of the line $x=x_{0}, y=y_{0}$. 
To show the necessity of the assumption of an order by order construction in Proposition 5, we present now a second power series solution which violates it. This yields a representation of the general solution using only one arbitrary function of one variable. It arises from the following initial value problem:

$$
u\left(t_{0}, x, y_{0}\right)=f(x) .
$$

Using the system in its original form (9), we can rewrite each derivative as a pure $x$-derivative

$$
\frac{\partial^{p+q+r} u}{\partial t^{p} \partial y^{q} \partial x^{r}}=\frac{\partial^{3 p+2 q+r} u}{\partial x^{3 p+2 q+r}}
$$

and thus express all Taylor coefficients by derivatives of $f$. Note, however, the crucial difference to the above constructed power series. The order of the derivative becomes greater in (25). Thus the corresponding power series cannot be constructed order by order and we cannot invoke Proposition 5 .

Whether or not a power series solution can be constructed order by order is not a purely technical point but has some importance for the convergence of the series. In Janet-Riquier theory (see Section 6) one can prove that for initial value problems where the power series solution can be obtained order by order the series converges in a neighborhood of the initial curve for arbitrary analytic Cauchy data. In problems where this is not the case additional conditions on the Cauchy data are usually necessary.

For $t_{0}=y_{0}=0$ one can find an integral representation of the general solution for this initial value problem using Fourier transform methods [1]:

$$
u(t, x, y)=\frac{\mathrm{e}^{2 y^{3} / 27 t^{2}}}{(3 t)^{1 / 3}} \int_{-\infty}^{\infty} \operatorname{Ai}\left(\frac{3 t(\xi-x)+y^{2}}{(3 t)^{4 / 3}}\right) \mathrm{e}^{y(\xi-x) / 3 t} f(\xi) \mathrm{d} \xi
$$

where $\operatorname{Ai}(z)$ denotes the Airy function. The invariance of the system under arbitrary translations of the independent variables allows one to extend this solution to $t_{0}, y_{0} \neq 0$. Obviously, this representation is not valid for arbitrary $f$, as the integral will converge only for functions $f$ falling rapidly enough as $x$ approaches infinity.

In the next section, we will also need the solution of the initial value problem where initial data is posed along parallels to the $y$-axis. There exist two possibilities. The first is:

$$
u\left(t_{0}, x_{0}, y\right)=f(y), \quad u_{x}\left(t_{0}, x_{0}, y\right)=g(y) .
$$

This time we have to consider all derivatives of the form

$$
u_{\{p q\}}:=\frac{\partial^{p+q} u}{\partial x^{p} \partial t^{q}} .
$$

As above we find that 
1. $u_{\{2 m, 0\}}=\partial^{m} u / \partial y^{m}$.

2. $u_{\{2 m+1,0\}}=\partial^{m} u_{x} / \partial y^{m}$.

3. $u_{\{p q\}}=\partial^{q} u_{\{p+q, 0\}} / \partial y^{q}$.

We see that the third case leads to an increase of the order. Thus it is no contradiction of Proposition 5 that (27) contains only two free functions of one variable.

The second possibility of an initial value problem along the $y$-axis is given by

$$
u\left(t_{0}, x_{0}, y\right)=f(y), \quad u_{t}\left(t_{0}, x_{0}, y\right)=g(y), \quad u_{x}\left(t_{0}, x_{0}, y_{0}\right)=a .
$$

We get exactly the same three cases as above with the only difference that in Case $2 \mathrm{~m}$ must be greater than 0 which leads to the additional constant.

\section{A General Cauchy Problem}

In this section we shall be guided by the results of Section 4 (which, in turn, depend on Propositions 4 and 5) in order to solve a general Cauchy problem for system (9). In the process we explicitly compute the characteristics for the system in terms of which the solution of the Cauchy problem either does not exist or, if it does, fails to be unique.

THEOREM 6. Let, be an arbitrary analytic curve in the $t-x-y$ space which is at no point tangent to a characteristic curve. Let $(\vec{t}, \vec{n}, \vec{b})$ denote unit tangent, normal and binormal vector along, , i.e. a moving orthogonal frame. If $u, \partial u / \partial \vec{n}, \partial u / \partial \vec{b}$ are analytic functions on, , then there exists locally one and only one analytic solution to the initial value problem

$$
\frac{\partial u}{\partial t}=\frac{\partial^{3} u}{\partial x^{3}}, \quad \frac{\partial u}{\partial y}=\frac{\partial^{2} u}{\partial x^{2}}
$$

$$
u, \partial u / \partial \vec{n}, \partial u / \partial \vec{b} \text { prescribed on }
$$

Proof. Since, is an analytic curve in $t-x-y$ space, there exists two real-valued, analytic functions $\eta(t, x, y)$ and $\zeta(t, x, y)$ such that, is described by $\eta=\zeta=0$. Since $d \eta \wedge d \zeta \neq 0$ generically, we can find a third analytic function $\xi(t, x, y)$ such that $d \xi \wedge d \eta \wedge d \zeta \neq 0$ generically. Now since $u, \partial u / \partial \vec{n}$ and $\partial u / \partial \vec{b}$ are prescribed and analytic along, , we can solve the linear system

$$
\left.\vec{t} \cdot \vec{\nabla} u\right|_{\Gamma}=\left.\frac{\partial u}{\partial \xi}\right|_{\Gamma},\left.\quad \vec{n} \cdot \vec{\nabla} u\right|_{\Gamma}=\left.\frac{\partial u}{\partial \vec{n}}\right|_{\Gamma},\left.\quad \vec{b} \cdot \vec{\nabla} u\right|_{\Gamma}=\left.\frac{\partial u}{\partial \vec{b}}\right|_{\Gamma}
$$

uniquely for $\left.\frac{\partial u}{\partial t}\right|_{\Gamma},\left.\frac{\partial u}{\partial x}\right|_{\Gamma},\left.\frac{\partial u}{\partial y}\right|_{\Gamma}$ (the uniqueness follows from the fact that $(\vec{t}, \vec{n}, \vec{b})$ forms an orthogonal frame along, ) where $\xi$ is the chosen parameter along, . 
Since the triad $(\xi, \eta, \zeta)$ forms a new coordinate system in a neighbourhood containing the curve, , a simple chain rule argument shows that the functions $\bar{u}(\xi, 0,0), \partial \bar{u} / \partial \eta(\xi, 0,0)$ and $\partial \bar{u} / \partial \zeta(\xi, 0,0)$ are uniquely determined and analytic along, , where we have introduced $\bar{u}(\xi, \eta, \zeta)=u(t, x, y)$.

To construct a formal power series solution order by order, we must use the involutive system (17) instead of the original system (9). We now transform our initial value problem (30) to this new coordinate system to obtain the following one expressed in matrix form

$$
\begin{gathered}
\left(\begin{array}{ccc}
\eta_{x}^{2} & \zeta_{x}^{2} & 2 \eta_{x} \zeta_{x} \\
\eta_{x} \eta_{y} & \eta_{x} \zeta_{y}+\eta_{y} \zeta_{x} & \zeta_{x} \zeta_{y} \\
\eta_{y}^{2}-\eta_{x} \eta_{t} & 2 \eta_{y} \zeta_{y}-\eta_{x} \zeta_{t}-\eta_{t} \zeta_{x} & \zeta_{y}^{2}-\zeta_{x} \zeta_{t}
\end{array}\right) \cdot\left(\begin{array}{c}
\frac{\partial^{2} \bar{u}}{\partial \eta^{2}} \\
\frac{\partial^{2} \bar{u}}{\partial \eta \partial \zeta} \\
\frac{\partial^{2} \bar{u}}{\partial \zeta^{2}}
\end{array}\right)=\left(\begin{array}{c}
T_{1} \\
T_{2} \\
T_{3}
\end{array}\right), \\
\bar{u}(\xi, 0,0), \frac{\partial \bar{u}}{\partial \eta}(\xi, 0,0), \frac{\partial \bar{u}}{\partial \zeta}(\xi, 0,0), \text { prescribed and analytic }
\end{gathered}
$$

where the terms $T_{1}, T_{2}, T_{3}$ on the right hand side involve $\xi, \eta, \zeta$ and their derivatives together with $\xi$-derivatives of $\bar{u}, \partial \bar{u} / \partial \eta, \partial \bar{u} / \partial \zeta$.

We prove the existence and uniqueness of a solution by constructing a power series solution. In Section 2 it was pointed out, that an order by order construction is only possible, if the system is involutive. On the other hand, we noted that involution is an intrinsic property of the submanifold $\mathcal{R}_{q}$ and basically independent of the coordinate system. Thus a problem can only arise, if our new coordinate system $(\xi, \eta, \zeta)$ is not $\delta$-regular.

We can now apply the criterion for an involutive symbol in reverse. We know, the system and thus its symbol is involutive. Hence the coordinate system is $\delta$ regular, if and only if the number of multiplicative variables is 7 as obtained in Section 3 for the original coordinate system. This will be the case only, if the matrix $M$ in the new system (32) is nonsingular. Then we have to take either $\eta$ or $\zeta$ as $x^{3}$ (depending on the row echelon form), the other one as $x^{2}$ and $\xi$ as $x^{1}$. This ordering leads to one equation of class 3 and two equations of class 2 and thus to a total of 7 multiplicative variables.

We have now transformed the original initial value problem into a form, where one can apply the Cartan-Kähler theorem $[7,16]$. It states the existence and uniqueness of the analytic solution for analytic involutive systems. Indeed, we know that we can uniquely construct a formal power series solution and by repeated application of the Cauchy-Kowalewsky theorem one can show that this series will converge in a neighbourhood of , .

In summary, we have shown that provided, is nowhere tangent to the family of curves

$$
\begin{gathered}
\eta(t, x, y)=\alpha, \quad \zeta(t, x, y)=\beta, \\
\operatorname{det} M=0
\end{gathered}
$$


then the solution of the Cauchy problem (30) will exist, be unique and analytic in a neighbourhood of ,. We complete the proof by noting that the family of curves defined by $(33,34)$ describes the complete set of characteristic curves for the initial value problem.

By direct inspection of the characteristic equation (34) we find at once two sets of solutions, namely

$$
\eta(t, x, y)=f_{1}(t, y), \quad \zeta(t, x, y)=f_{2}(t, y)
$$

and

$$
\eta(t, x, y)=g_{1}(t, x), \quad \zeta(t, x, y)=g_{2}(t, x)
$$

where the functions $f_{1}, f_{2}, g_{1}, g_{2}$ are differentiable but otherwise completely arbitrary.

Now turning to the curves defined by (33), we see that (35) leads to the equation

$$
f_{1}(t, y)=\alpha, \quad f_{2}(t, y)=\beta \text {. }
$$

Since $f_{1}, f_{2}, \alpha, \beta$ are arbitrary, (37) describes an arbitrary straight line parallel to the $x$-axis in $x-y$-t space. This specifies one family of characteristic curves for our problem. Similar remarks using the solution (36) show that arbitrary straight lines parallel to the $y$-axis define a second family of characteristic curves. Since the characteristic equation (34) is underdetermined, there exists, however, further solutions than the two above mentioned.

We note that if initial data is prescribed along characteristic curves, then less Cauchy data than specified in Theorem 6 is required. In the previous section, we gave explicit power series solutions for the two families of characteristics found above. However, these cannot be constructed order by order.

Finally, we can deduce from Theorem 6 and the results of Refs. [1, 2] the following

COROLLARY 7. There exists a solution of the $(2+1)$-dimensional Krichever-Novikov equation (1) in a tubular neighborhood of any analytic curve, in $(x-y-t)$ space whenever, is not a characteristic curve as defined in Theorem 6 .

\section{Janet-Riquier Theory}

The present formal analysis of the system (9) can also be used to demonstrate some differences between the notion of involution in the formal theory and the notion of passivity in the Janet-Riquier theory which is based on differential algebra. Passive systems are often also called involutive, they are, however, in general only 
formally integrable. We will not explain this approach but refer the reader to the literature $[9,10]$.

As already mentioned, in formal theory a differential equation is defined as a fibred submanifold in a jet bundle. Involution is a property of this geometric object and is independent of the specific coordinate system or set of equations used to describe it. In Janet-Riquier theory, however, it is required to specify a ranking for the derivatives in order to define passivity. This still holds, even when we restrict ourselves to orderly rankings, i.e. when we always order a higher order derivative before a lower order derivative.

We can demonstrate this readily using system (17) and the standard form algorithm of Reid [17]. Within the framework of formal theory (17) (considered as a submanifold of $\mathcal{J}_{2}$ ) is an involutive system. With respect to the orderly lexicographic ordering induced by $t>y>x$ the system is however not passive within Janet-Riquier theory. We must add one more equation: $u_{y y y}=u_{t t}$.

That this equation is indeed necessary, can easily be seen, if we apply the initial data algorithm of Reid [17]. This algorithm generates a formally well-posed initial value problem [13] for a given passive system, that allows one to construct the general solution of the system. Reid uses this information to calculate the arbitrariness of the solution space.

This algorithm can most easily be understood using a graphic representation. Figure 1 shows the Reid diagram for the heat equation $u_{x x}-u_{y}=0$ using an orderly ranking of the derivatives. The axes represent the independent variables, the dots the different derivatives of $u$. The circle around $u_{x x}$ indicates that it is the leading derivative of the equation. All derivatives in the dashed area can be obtained from it by differentiating the equation, i.e. they are principal derivatives. All other derivatives are parametric. They must be prescribed by initial data. As indicated by the two small arrows, they can be ordered into two straight lines parallel to the $y$-axis. Thus we must prescribe $u$ and $u_{x}$ for $x=$ const to obtain a unique solution.

Figure 2 draws the same diagram for the full system (17) which is involutive in the sense of formal theory using the orderly ranking induced by $t>y>x$. Since there are now three independent variables, the picture is three-dimensional and we omit the depiction of the individual derivatives by dots. One can, however, clearly recognize, that the $(t-y)$-plane contains only parametric derivatives. The only further parametric derivative is $u_{x}$. Thus Reid's initial data algorithm leads to the following initial value problem, containing one free constant and one free function of two variables:

$$
u\left(t, x_{0}, y\right)=f(t, y), \quad u_{x}\left(t_{0}, x_{0}, y_{0}\right)=a .
$$

Seemingly, this violates Proposition 5. But a closer look at the prolongation $\mathcal{R}_{3}^{(1)}$ reveals that the function $f$ can not be chosen arbitrarily but must satisfy the differential equation $f_{t t}=f_{y y y}$. Using the same methods as in the proof of Proposition 5 , 


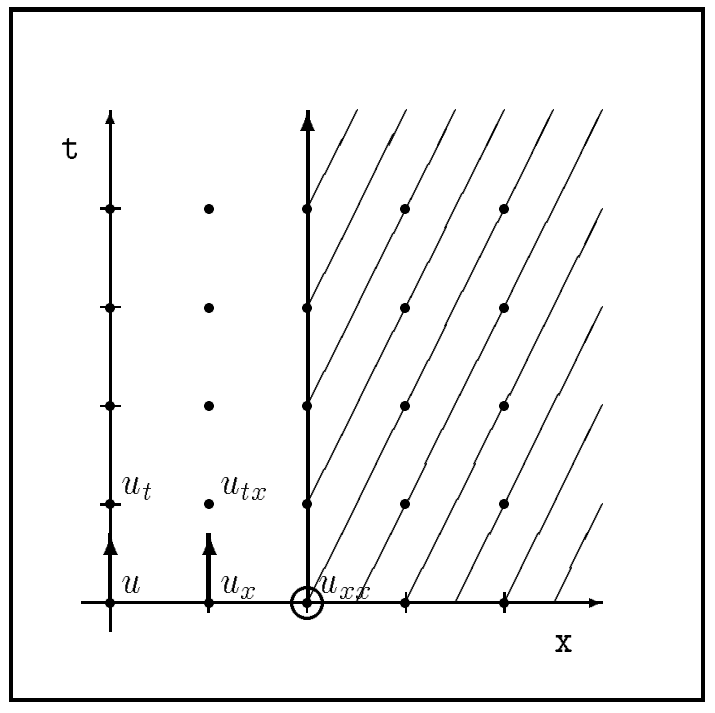

Fig. 1. Reid diagram for heat equation.

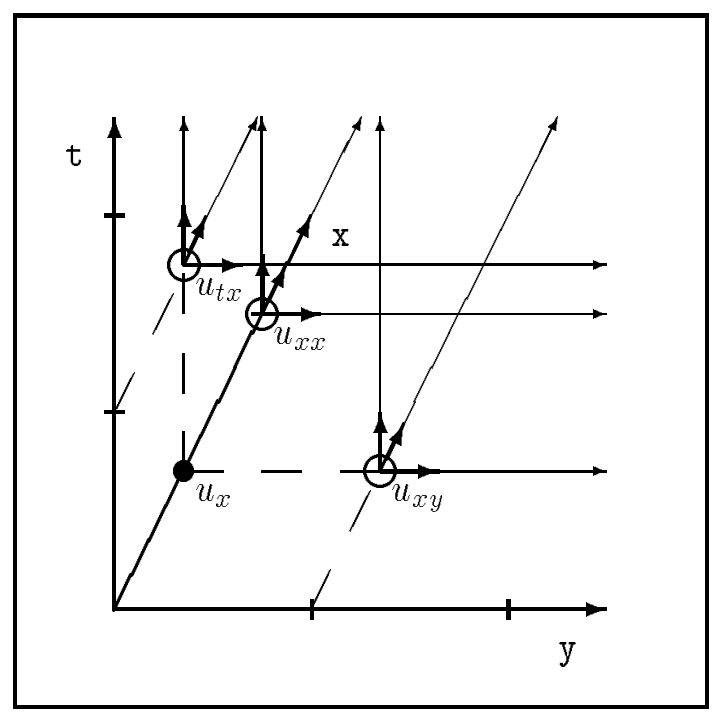

Fig. 2. Reid diagram for system (17). 
one can show that the solution space of this equation is spanned by three functions each of one variable!

But if we first apply the standard form algorithm to system (17) to make it passive in the sense of Janet-Riquier and then the initial data algorithm, we will get a correct result, as the standard form includes the equation $u_{y y y}-u_{t t}=0$ which leads of course to the above condition on the function $f$. Reid's algorithm yields now the initial value problem

$$
\begin{gathered}
u\left(t, x_{0}, y_{0}\right)=f(t), \quad u_{y}\left(t, x_{0}, y_{0}\right)=f_{1}(t), \quad u_{y y}\left(t, x_{0}, y_{0}\right)=f_{2}(t), \\
u_{x}\left(t_{0}, x_{0}, y_{0}\right)=a .
\end{gathered}
$$

The apparent contradiction stemmed only from the incorrect application of the initial data algorithm to a system which was not passive.

The effect that further equations have to be added to an already involutive system to render it passive is not yet well understood. One could conjecture that it is connected with the fact that the ordering $t>y>x$ does not lead to a $\delta$-regular coordinate system.

Finally, one should note that, if we restrict ourselves to orderly rankings, the two initial value problems (21) and (39) are the only possibilities, as the only freedom lies in the ordering of the third equation of system (17).

The reason for the importance of orderly rankings lies in the fact that only for them Riquier's theorem [18] guarantees the existence and uniqueness of an analytic solution. Indeed, we have seen that in case of the initial value problem (24) additional conditions had to be posed on the initial data in order to prove the convergence of the power series.

\section{Acknowledgements}

CR acknowledges with gratitude support under an ARC grant. WMS wishes to thank J.F. Pommaret and G. Reid for many helpful discussions. He is supported by Studienstiftung des deutschen Volkes. PJV thanks I. Lisle for early discussions on various aspects of the problems dealt with in this paper and especially discussion of the Reid-Lisle Initial Data Algorithm. We acknowledge with gratitude the hospitality of the Centre de Recherches Mathématiques in Montréal where part of the research for this paper was carried out.

\section{References}

1. C. Rogers, W. Oevel, and P.J. Vassiliou. Invariance under reciprocal transformations in $1+1$ and $2+1$ dimensions. In Proc. Int. Meeting on Nonlinear Diffusion Phenomena, Bangalore, 1992. Indian Institute of Science. 
2. C. Rogers, M.P. Stallybrass, and P.J. Vassiliou. An invariance of the $(2+1)$-dimensional Harry-Dym equation: Application to an initial/boundary value problem. Int. J. Nonlin. Mech., 29:145-154, 1994.

3. C. Rogers. The Harry-Dym equation in $2+1$ dimensions: A reciprocal link with the Kadomtsev-Petviashvili equation. Phys. Lett., A120:15-18, 1987.

4. W. Oevel and C. Rogers. Gauge transformations and reciprocal links in $2+1$ dimensions. Rev. Math. Phys., 5:299-330, 1993.

5. B.G. Konopelchenko and V.G. Dubrovsky. Some new integrable nonlinear evolution equations in 2+1 dimensions. Phys. Lett., A102:15-17, 1981.

6. C. Rogers, M.P. Stallybrass, and W.K. Schief. Initial/boundary value problems and Darboux-Levi type transformations associated with a (2+1)-dimensional eigenfunction equation. Int. J. Nonlin. Mech., 30:223-233, 1995.

7. J.F. Pommaret. Systems of Partial Differential Equations and Lie Pseudogroups. Gordon \& Breach, London, 1978.

8. W.M. Seiler. On the arbitrariness of the general solution of an involutive partial differential equation. J. Math. Phys., 35:486-498, 1994.

9. M. Janet. Sur les Systèmes d'Équations aux Dérivées Partielles. J. Math. Pure Appl., 3:65-151, 1920.

10. C. Riquier. Les Systèmes d'Équations aux Derivées Partielles. Gauthier-Villars, Paris, 1910.

11. W.M. Seiler. Generalized tableaux and formally well-posed initial value problems. Preprint Lancaster University, 1995.

12. D.J. Saunders. The Geometry of Jet Bundles. London Mathematical Society Lecture Notes Series 142. Cambridge University Press, Cambridge, 1989.

13. W.M. Seiler. Analysis and Application of the Formal Theory of Partial Differential Equations. PhD thesis, School of Physics and Materials, Lancaster University, 1994.

14. J. Schü, W.M. Seiler, and J. Calmet. Algorithmic methods for Lie pseudogroups. In N. Ibragimov, M. Torrisi, and A. Valenti, editors, Proc. Modern Group Analysis: Advanced Analytical and Computational Methods in Mathematical Physics, pages 337-344, Acireale (Italy), 1992. Kluwer, Dordrecht 1993.

15. W.M. Seiler. Applying AXIOM to partial differential equations. Internal Report 95-17, Universität Karlsruhe, Fakultät für Informatik, 1995.

16. H.L. Goldschmidt. Integrability criteria for systems of non-linear partial differential equations. J. Diff. Geom., 1:269-307, 1969.

17. G.J. Reid. Algorithms for reducing a system of PDEs to standard form, determining the dimension of its solution space and calculating its Taylor series solution. Eur. J. Appl. Math., 2:293-318, 1991.

18. J.M. Thomas. Riquier's existence theorems. Ann. Math., 30:285-321, 1929. 